Conclusions CT and NG infections are associated with positive HIV status and other STI coinfections. We confirmed the association between NG and CT and several known risk factors of the index patient; surprisingly, none of the partnership factors (eg, concurrency, meeting place, or partner's HIV status), except age difference, were associated with NG or CT.

\section{P1-S2.50 ANAL NON-L CHLAMYDIA TRACHOMATIS INFECTION IN MEN WHO HAVE SEX WITH MEN, AT L'ACTUEL, MONTREAL, QUEBEC}

doi:10.1136/sextrans-2011-050108.107

L Charest, R Thomas, B Trottier, M Boissonnault, D Legault, D Longpré, J Szabo, P J Maziade, N Machouf. Clinique médicale l'Actuel, Montréal, Canada

Few cases of sexually transmitted Hepatitis C infection (HCV) in men who have sex with men (MSM) had been reported in the literature.

Background The incidence of genital infection from non-L Chlamydia trachomatis (CT) is increasing in the province of Quebec. Traditionally it was more prevalent in young women. In the last few years, CT infection has emerged in men who have sex with men (MSM) group. The objective of this study was to describe anal nonL CT infection in MSM in an HIV-STI clinic in a large urban setting. Methods Retrospective study of anal non-L CT cases diagnosed at Clinique l'Actuel in 2010. We collected information on age at diagnosis, HIV status, CT serovars, symptoms, mode of transmission and concomitant presence of CT in urine. An anal swab specimen was collected with a TAAN test (BD Probe Tec TM).

Results From 2006 to 2009, we diagnosed respectively 42, 58, 82 and 118 anal non-L CT infection in MSM. In 2010, we diagnosed 154 different anal non-L CT infections in 152 MSM. Patient's age at diagnosis was 33 years (IOR-25-38) from 18 to 70 years. 28\% were HIV+ at time of diagnosis. Anal infection was primarily caused by serovars J (35\%), G (31\%), D (23\%), E (10\%), F (1\%) and C (1\%). Among HIV patients the serovars was predominantly J (46\%), D $(30 \%)$ and G (24\%). But this difference was not statistically significant. Anal non-L CT infection was detected in asymptomatic patients while screening in $54 \%$ of case. $7 \%$ of MSM had no symptom but consulted because they were notified by a sexual partner. $39 \%$ had symptoms-proctitis (26\%), urethritis (11\%) or symptom of other STI including syphilis and HIV primary infection $(2 \%)$. Mode of transmission for the anal infection was not specifically specified in the file of the patients, but $88 \%$ reported anal unprotected penetration in the past year, $12 \%$ did not report anal penetration but had mouth to anus contact (rimming) or friction from penis to anus. One patient reports sharing anal toys. Urine CT was positive in $18 \%$ of MSM, so those MSM had two infected sites. Conclusion Given the high rate of positive results, MSM should be screen with a TAAN test for anal CT infection. This screening should be included in the regular follow-up of HIV patients. MSM community should be aware of this emerging infection. Public health authority should focus on developing and promoting new guidelines of screening, access to validated test with identification of serovars.

\section{P1-S2.51 SEXUALLY TRANSMITTED HCV IN MSM IN MONTREAL}

doi:10.1136/sextrans-2011-050108.108

R Thomas, S Vezina, N Machouf, R O'Brien, E Huchet, D Longpré, S Lavoie, M Milne, F Asselin, B Trottier. Clinique médicale l'Actuel, Montreal, Canada

Methods A retrospective analysis of the clients who attend the "Gay Screening Clinic" (GSC) at Clinique medical l'Actuel (Montreal, Canada) between May 2009 and April 2010 was conducted.
Results Among the 1010 patients included in the analyses we found five cases of new HCV infection which seems to be acquired by sexual transmission. All of them were male and MSM, none of the cases had previous history of IDU, but three reported having had sexual relation with IDU partners. All of them were previously vaccinated for Hepatitis $A$ and $B$ and screened for HIV. None of them were coinfected with HIV, but one was coinfected with Syphilis and one with Gonorrhoea. Patients infected with HCV were older than non HCV patients ( 37 years vs 32 years) and had a higher number of sexual partners during the last 12 months (16 part. vs 9 for the non HCV patients). One of the HCV cases reported not having anal intercourse, two reported having had occasional unprotected anal intercourse and two reported always using condom for anal intercourse.

Conclusions As in other urban centers, cases of sexually transmitted $\mathrm{HCV}$ had also been found in Montreal. This prevalence of $0.5 \%$ is very low compared to our HIV population in which the prevalence of sexually transmitted HCV is $3 \%$. The particularity of MSM with recently acquired HCV by sexual transmission seems to be related to their engagement in sexual relation with high number of concurrent partners.

\section{P1-S2.52 RISK FACTORS FOR STIS AMONG MSM ATTENDING A SEXUALLY TRANSMITTED DISEASE CLINIC IN MONTREAL, CANADA}

doi:10.1136/sextrans-2011-050108.109

N Machouf, R Thomas, R O’Brien, S Vezina, D Longpré, D Legault, M Milne, J Fafard, B Trottier. Clinique médicale l'Actuel, Montreal, Canada

Background Often STIs are not diagnosed and not treated because people don't have access to appropriate healthcare screening facilities and to care. At Clinique l'Actuel (Montreal, Canada) we developed Gay Screen Clinic (GSC) as a new concept giving rapid access to men who have sex with men (MSM) to an appointment for STI screening. We then assessed the extend and risk factors of STIs in a population of men having sex with men (MSM) attending the GSC.

Methods We did a retrospective analysis of the last 1000 attendees to the GSC at Clinique l'Actuel in 2009-2010. Multivariable analyses were conducted to identify the factors associated with history of STIs.

Results Participants were all MSM with a mean age of 32 years (ranged from 18 to $70 \mathrm{y})$. In total, $50 \%(n=506)$ of them self reported history of STIs and $236(24 \%)$ of them had a positive sexual health screen at this visit. STI diagnoses included genital herpes $(n=105,14 \%)$, condylomes $(n=79,8 \%)$, syphilis $(n=43,5 \%)$, chlamydia infection $(n=32,3 \%), \operatorname{HIV}(n=10,1 \%)$, Gonorrhoea $(n=9,1 \%)$ and $\operatorname{HCV}(n=5,1 \%) .32 \%$ of the attendees had sexual relations in bath houses and $43 \%$ with anonymous contacts. In multivariate analyses, past history of STI was significantly associated with higher age $(O R=1.02, p=0.001)$, higher number of sexual partners in the last 12 months $(O R=1.02, p=0.015)$, having sexual contact in bath houses $(O R=1.46, p=0.021)$ and with unknown partners met through internet or in backrooms $(\mathrm{OR}=1.53, \mathrm{p}=0.004)$, using recreational drugs $(\mathrm{OR}=2.01, \mathrm{p}=0.001)$ and having only male partners $(O R=1.60, p=0.023)$ rather than male and female sexual partners.

Conclusions STIs were common among non HIV MSM attending the GSC in Montreal. Even after many years of prevention campaign MSM still have high risk sexual behaviour. Physician should routinely enquire about drug use of their patients in order to prevent new STIs. Targeting specific sexual networks is needed to be more effective. 